\title{
Underlay versus Interweaved Cognitive Radio Networks: a Performance Comparison Study
}

\author{
Miltiades C. Filippou ${ }^{1}$, David Gesbert ${ }^{1}$ and George A. Ropokis ${ }^{1,2}$ \\ ${ }^{1}$ Mobile Communications Department, EURECOM, 06410, Biot, France \\ ${ }^{2}$ Computer Technology Institute and Press "Diophantus", 26500, Rio-Patras, Greece \\ \{filippou,gesbert,ropokis\}@eurecom.fr
}

\begin{abstract}
In this paper, an analytical performance study is carried out for cognitive radio (CR) systems with the intent of comparing the two most popular CR designs, namely the interweaved and underlay setups. The comparison is carried out on an equal footing, by measuring the achievable ergodic capacity for secondary communication, under a common constraint on primary user's (PU) communication disturbance. Closed form expressions for the outage probability at the PU as well as expressions for the ergodic capacity of the secondary user (SU) are derived, as a function of key system parameters, under a rate-optimal sensing protocol for the interweaved scenario, and a standard power control policy for the underlay setting. The analysis reveals the regimes (in terms of primary activity, interference tolerance levels at the PU) where the interweaved design outperforms the underlay one and reciprocally.

Index Terms-Cognitive radio, interweaved, underlay, outage probability, ergodic capacity.
\end{abstract}

\section{INTRODUCTION}

Cognitive radio networks (CRNs) have recently emerged as a novel, promising technology which aims to tackle the problem of spectrum scarcity and thus, to enhance spectral efficiency via enabling better utilization of the currently underutilized radio spectrum [1], [2]. So far, two of the most popular approaches for CRN design have been proposed: (i) underlay (or spectrum sharing) CRNs, where a primary service provider allows the concurrent use of its spectral resources by an unlicensed secondary system, provided that the harmful interference generated by the secondary transmitter will not overcome a specified maximum tolerated level and (ii) interweaved (or opportunistic) CRNs, in which the secondary network (either the transmitter or the receiver) senses the frequency spectrum and utilizes the channels when they are not occupied by primary transmissions.

To the best of our knowledge, little attempt has been made to compare such designs on an equal footing, and even less so on an analytical basis. Indeed, the philosophies behind each alternative seem irreconcilable at first glance: the underlay approach being typically reserved for applications with little or no quality of service (QoS) guarantees at the legacy (primary) network, while the interweaved design is expected to offer a near-zero disturbance at the PU hence offers hard QoS guarantees, or so it seems.

Upon closer examination, it is well known that the QoS offered at the PU in an interweaved scenario is only as good as the sensing capability at the secondary nodes. Sensing imperfections due to channel fading, shadowing, or noise give rise to miss-detection events, which, in turn, potentially lead to outage events at the PU due to the creation of unintentional interference towards it [3]. Conversely, although an over-conservative sensing design would make sure near zero interference is generated at the PU, it would also lead to an over-spending of secondary communication resources towards sensing resulting to a degraded rate at the secondary receiver. Hence, an interesting trade-off emerges between outage events at the PU and average (ergodic) rate performance at the SU. Interestingly, an underlay radio network can also be evaluated under the prism of this very same trade-off. In the underlay case, the power control policy at the secondary transmitter replaces sensing as the protocol capable of striking various points in the (PU outage, SU rate) region.

This observation motivates us to compare the performance of the interweaved and underlay CRN approaches under a common figure of merit. More specifically, we are interested in comparing the two CRN approaches in terms of the achievable SU ergodic rate subject to a common constraint on the PU outage probability.

Some interesting prior work is worth mentioning in this context. In [4], the throughput potential of different CR techniques has been investigated from an information theoretical point of view, however no expressions describing the ergodic capacity of the SU or the outage probability of the primary system are given considering a fading environment. Moreover, in [5], although expressions for the instantaneous rate of the SU are given, the rather unrealistic assumption of perfect spectrum sensing is adopted. Furthermore, in [6], [7] and [8] new spectrum sharing models are proposed, either mixed ones or variants of the interweaved model, though no straightforward performance comparison of the two mentioned CRN approaches is provided. On the other hand, works such as [9]-[14] emphasize on the derivation of either approximations or closed form expressions for the ergodic capacity of SU as well as for the outage probability of the primary system. Yet, no comparison between interweaved and underlay CRN approaches is illustrated in these works.

In this paper, both the interweaved and underlay CRN approaches are investigated with respect to a SISO network and compared with reference to the ergodic capacity of the 
SU for a target, common PU outage probability as well as for various primary communication activity profiles. More concretely, our contributions are the following:

- Closed form expressions for the outage probability of primary communication, regarding both interweaved and underlay approaches, are derived.

- Expressions for the ergodic capacity of the SU are derived with respect to both CRN approaches.

- The generic design parameters of each approach corresponding to a target PU outage probability are optimized with the aim of comparing the optimal throughput behavior of the two examined approaches.

- We finally compare the optimal SU ergodic throughput of the two CRN approaches under a target PU outage level for various primary communication activity profiles. An outage event is declared when the rate at the PU falls below a given threshold (whether due to interference or not). It is observed that the performance comparison results are related to the activity profile of primary communication.

Throughout the paper, the following notations are adopted: $\mathcal{P}(A)$ denotes the probability of event $\mathrm{A}$ and $\mathbb{E}\{\cdot\}$ symbolizes the expectation operator. For a scalar random variable $X, X \sim \mathcal{C N}\left(\mu, \sigma^{2}\right)$ denotes that $X$ follows a circularly symmetric complex Gaussian (CSCG) distribution with mean $\mu$ and variance $\sigma^{2}$. Also, $E_{1}(\cdot)$ represents the exponential integral function, which is defined in [15, 5.1.1], whereas $\mathcal{Q}(x)=\frac{1}{2} \operatorname{erfc}\left(\frac{x}{\sqrt{2}}\right)$ stands for the complementary Gaussian distribution function, where function $\operatorname{erfc}(\cdot)$ is defined in $[15$, 7.1.2].

\section{System And Channel Model}

The system under investigation, which is illustrated in Fig. 1 consists of a SISO primary system, comprising of a base station (BS), $B S_{p}$, as well as its assigned PU, $U_{p}$.

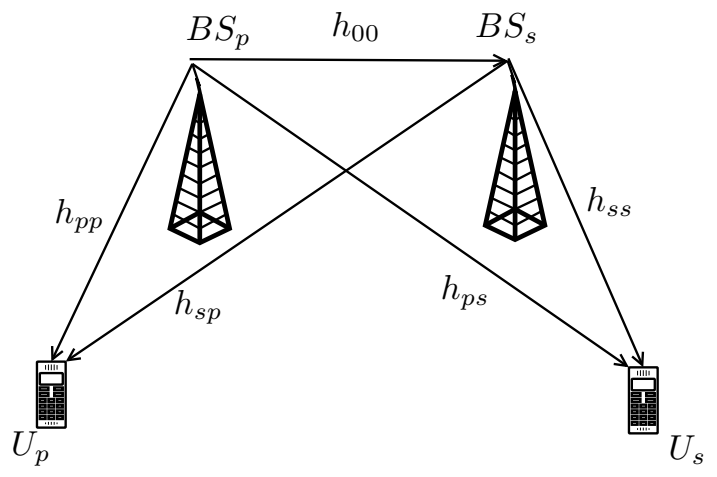

Fig. 1. Examined CRN topology.

Focusing on downlink communication, the primary system is willing to share its resources with a SISO secondary system consisting of a BS, $B S_{s}$, along with its assigned SU, $U_{s}$. It is assumed that channels $h_{i j}, \quad i, j \in\{p, s\}$ between $B S_{i}$ and user $U_{j}$ as well as channel $h_{00}$ between $B S_{p}$ and $B S_{s}$ are Rayleigh fading ones, i.e., $h_{i j} \sim \mathcal{C N}\left(0, \sigma_{i j}^{2}\right) \quad i, j \in\{p, s\}$ and $h_{00} \sim \mathcal{C N}\left(0, \sigma_{00}^{2}\right)$. Moreover, in the rest of the paper, channel power gains $\left|h_{i j}\right|^{2}, i, j \in\{p, s\}$ and $\left|h_{00}\right|^{2}$ will be denoted by $\gamma_{i j}$ and $\gamma_{00}$, respectively.

In the following two sections, closed form expressions for the outage probability of PU as well as expressions for the achievable ergodic capacity of SU will be derived for both CRN approaches.

\section{INTERWEAVED APPROACH}

\section{A. General model for interweaved CRNs}

Focusing on the interweaved approach, as Fig. 2 shows, each medium access control (MAC) frame has a duration of $T$ time units and consists of a slot dedicated to spectrum sensing which lasts for $\tau<T$ time units. The rest of the frame is dedicated to data transmission. Moreover, during each sensing phase, $B S_{s}$ receives $N=\tau f_{s}$ samples, where $f_{s}$ is the sampling frequency of the received signal. It is also assumed that during the sensing phase, $B S_{s}$ ceases any transmission and all instantaneous channels are fixed within a MAC frame. It should be noted that the spectrum sensing procedure is carried out at the secondary transmitter in order to avoid considering a feedback link from the $\mathrm{SU}$ to $B S_{s}$, merely dedicated to spectrum sensing decision signaling.

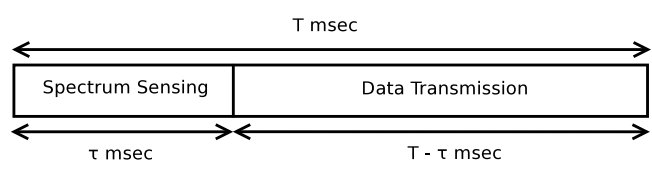

Fig. 2. MAC frame structure.

The binary hypothesis test for spectrum sensing at the n-th, $n=1,2, \ldots, N$ time instant is expressed as

$$
y_{s}[n]= \begin{cases}z[n], & \text { if } \mathcal{H}_{0} \\ \sqrt{P_{p}} h_{00} s_{p}[n]+z[n], & \text { if } \mathcal{H}_{1},\end{cases}
$$

where the additive noise $z[n]$ is a CSCG, independent, identically distributed (i.i.d) process with $z[n] \sim \mathcal{C N}\left(0, N_{0}\right), P_{p}$ is a fixed power level at $B S_{p}$ and the information symbol $s_{p}[n]$ is selected from a CSCG codebook, i.e., $s_{p}[n] \sim \mathcal{C N}(0,1)$ and is independent of $z[n]$. As a result, signal $s[n]=\sqrt{P_{p}} h_{00} s_{p}[n]$, for a fixed channel $h_{00}$, follows a CSCG distribution with zero mean and variance $\sigma_{s}^{2}=\mathbb{E}\left\{|s[n]|^{2}\right\}=P_{p} \gamma_{00}$.

Fixing a sensing time, $\tau$, as well as an energy detection threshold, $\epsilon$, by applying central limit theorem, one can calculate the false alarm probability, $\mathcal{P}_{f a}$, as well as the corresponding probability of detection, $\mathcal{P}_{d}$, with respect to a specific MAC frame, by applying [16, Proposition 1, Proposition 2]. The above probabilities are then written as

$$
\mathcal{P}_{f a}=\mathcal{Q}\left(\sqrt{N}\left(\frac{\epsilon}{N_{0}}-1\right)\right), \quad \mathcal{P}_{d}=\mathcal{Q}\left(\frac{\epsilon-\mu_{1}}{\sigma_{1}}\right)
$$

where $\mu_{1}=P_{p} \gamma_{00}+N_{0}$ and $\sigma_{1}^{2}=\frac{N_{0}^{2}}{N}\left(\frac{P_{p} \gamma_{00}}{N_{0}}+1\right)^{2}$.

In pursuance of deriving an average detection probability with reference to fading channel $h_{00}$, we have 


$$
\mathcal{P}_{d}^{a v g}=\int_{0}^{\infty} \mathcal{P}_{d}\left(\gamma_{00}\right) f_{\gamma_{00}}\left(\gamma_{00}\right) d \gamma_{00}
$$

However, since the exact form of the above expectation is a complicated function of generic parameters, $\tau$ and $\epsilon$, which need to be later optimized in terms of maximizing the ergodic throughput for the SU, we choose to apply a bound of this expectation. Detection probability, $\mathcal{P}_{d}$, is a concave function of its argument, thus, by applying Jensen's inequality, an upper bound for the expectation over fading can be extracted. This bound is the following

$$
\mathcal{P}_{d, B}=\mathcal{Q}\left(\sqrt{N}\left(\frac{\epsilon}{P_{p} \sigma_{00}^{2}+N_{0}}-1\right)\right) .
$$

Regarding the average false alarm probability, it remains the same under any fading channel, for given $\tau$ and $\epsilon$, since $\mathcal{P}_{f a}$ is considered for the case where no primary signal is transmitted, thus

$$
\mathcal{P}_{f a}^{a v g}=\mathcal{P}_{f a} .
$$

\section{B. Outage probability of primary communication}

An outage event is declared at the primary system when, given that the primary network is active, the SINR of the PU is below a predefined threshold, $\zeta_{0}$. This can occur in two cases: 1 . when a missed detection takes place at $B S_{s}$, potentially resulting to a PU SINR that is less than $\zeta_{0}$ or 2 . when the secondary system has accurately detected the presence of a primary signal and halts transmission, however, the desired signal received at the PU is not strong enough in order for the SINR of PU to overcome threshold $\zeta_{0}$. In the following proposition, a closed form expression of PU outage probability is given.

Proposition 1. The outage probability of primary communication for an interweaved $\mathrm{CRN}$ is given by the following expression

$$
\mathcal{P}_{\text {out }}^{\text {int }}=1-\left(\frac{\alpha_{1}+\alpha_{2} \mathcal{P}_{d}}{\alpha_{1}+\alpha_{2}}\right) e^{\frac{-\zeta_{0} N_{0} B}{\alpha_{1}}},
$$

where $\alpha_{1}=\sigma_{p p}^{2} P_{p}, \alpha_{2}=\sigma_{s p}^{2} P_{s} \zeta_{0}$ and $P_{s}$ expresses the maximum instantaneous available power at the secondary transmitter.

Proof. A detailed proof is provided in [17].

\section{Ergodic capacity of secondary communication}

Using upper bound $\mathcal{P}_{d, B}$, instead of average detection probability $\mathcal{P}_{d}^{a v g}$, the ergodic rate of the SU is lower bounded by

$$
\begin{aligned}
& \mathbb{E}\left\{R_{s}^{\text {int }}\right\} \geq \frac{(T-\tau)}{T}\left(\mathcal{P}\left(\mathcal{H}_{0}\right)\left(1-\mathcal{P}_{f a}\right) B \mathbb{E}\{\underbrace{\log \left(1+\frac{P_{s} \gamma_{s s}}{N_{0} B}\right)}_{\mathcal{A}_{1}}\}\right. \\
& \left.+\mathcal{P}\left(\mathcal{H}_{1}\right)\left(1-\mathcal{P}_{d, B}\right) B \mathbb{E}\{\underbrace{\log _{2}\left(1+\frac{P_{s} \gamma_{s s}}{N_{0} B+P_{p} \gamma_{p s}}\right)}_{\mathcal{A}_{2}}\}\right) .
\end{aligned}
$$

With the aim of computing the expectation appearing in the first term of (7), we observe that random variable $\frac{P_{s} \gamma_{s s}}{N_{0} B}$ is an exponentially distributed random variable. Thus, using $[15$, 5.1.28] we have

$$
\mathbb{E}\left\{\mathcal{A}_{1}\right\}=\frac{1}{\ln (2)} e^{\frac{1}{\bar{\gamma}}} E_{1}\left(\frac{1}{\bar{\gamma}}\right)
$$

where $\bar{\gamma}=\frac{P_{s} \sigma_{s s}^{2}}{N_{0} B}$. For the expectation appearing in the second term of (7), as random variables $P_{s} \gamma_{s s}$ and $P_{p} \gamma_{p s}$ are exponentially distributed, by making use of $[15,5.1 .28]$, one obtains

$$
\begin{aligned}
\mathbb{E}\left\{\mathcal{A}_{2}\right\} & =\frac{1}{\ln (2)}\left(\frac{\bar{x}_{1}}{\bar{x}_{1}-\bar{x}_{2}}\right) e^{\frac{N_{0} B}{\bar{x}_{1}}} \times \\
& \left(E_{1}\left(\frac{N_{0} B}{\bar{x}_{1}}\right)-e^{N_{0} B\left(\frac{\bar{x}_{1}-\bar{x}_{2}}{\bar{x}_{1} \bar{x}_{2}}\right)} E_{1}\left(\frac{N_{0} B}{\bar{x}_{2}}\right)\right),
\end{aligned}
$$

where $\bar{x}_{1}=P_{s} \sigma_{s s}^{2}, \bar{x}_{2}=P_{p} \sigma_{p s}^{2}$ and it is assumed that $\bar{x}_{1}>\bar{x}_{2}$ or equivalently, $\sigma_{s s}^{2}>\sigma_{p s}^{2}$ for a CRN with $P_{s}=P_{p}$.

\section{UNDERLAY APPROACH}

\section{A. General model for underlay CRNs}

In the underlay CRN approach, the primary system allows the reuse of its spectral resources by the newcoming secondary system subject to a constraint considering a maximum tolerated interference power level at the PU, denoted by $\mathcal{I}$. Let $P_{s}^{u n d}$ be the transmitted power by $B S_{s}$, which can vary from zero to a maximum instantaneous value, $P_{s}$. The maximum instantaneous power level is taken to be equal to the maximum instantaneous power level at $B S_{s}$ for the interweaved approach in order to realize a fair comparison from a power consumption perspective. A truncated power transmission policy will be applied in this case. This policy is the following

$$
P_{s}^{\text {und }}= \begin{cases}\frac{\mathcal{I}}{\gamma s p}, & \text { if } \frac{\mathcal{I}}{\gamma_{s p}}<P_{s} \\ P_{s}, & \text { if } \frac{\mathcal{I}}{\gamma_{s p}} \geq P_{s} .\end{cases}
$$

In the following, a closed form expression for the outage probability of the PU as well as an approximation for the achievable ergodic capacity of SU will be derived, focusing on the underlay approach.

\section{B. Outage probability of primary communication}

The primary system is in outage when the instantaneous SINR at the PU is below threshold $\zeta_{0}$. In the proposition that follows, a closed form expression of PU outage probability is given.

Proposition 2. The outage probability of primary communication for an underlay $\mathrm{CRN}$ is given by

$$
\begin{array}{r}
\mathcal{P}_{\text {out }}^{\text {und }}=e^{-\frac{\mathcal{I}}{P_{s} \sigma_{s p}^{2}}}\left(1-e^{-\frac{\zeta_{0}\left(N_{0} B+\mathcal{I}\right)}{\alpha_{1}}}\right)+\frac{1}{P_{p} \sigma_{s p}^{2} \sigma_{p p}^{2} \delta_{2}} \times \\
\left(e^{-\delta_{2} \zeta_{0} N_{0} B} \frac{1}{\delta_{1}}\left(e^{-\frac{\mathcal{I} \delta_{1}}{P_{s}}}-1\right)+\sigma_{s p}^{2}\left(1-e^{-\frac{\mathcal{I}}{P_{s} \sigma_{s p}^{2}}}\right)\right),
\end{array}
$$


where $\delta_{1}=\frac{1}{\sigma_{s p}^{2}}+\frac{P_{s} \zeta_{0}}{\alpha_{1}}$ and $\delta_{2}=\frac{1}{\alpha_{1}}$.

Proof. A detailed proof is provided in [17].

\section{Ergodic capacity of secondary communication}

Following the power transmission policy described in (10), the expression describing the ergodic rate of the SU will be the one that follows

$$
\begin{aligned}
\mathbb{E}\left\{R_{s}^{u n d}\right\} & =\mathcal{P}\left(\mathcal{H}_{0}\right) B \mathbb{E}\{\underbrace{\log \left(1+\frac{P_{s}^{u n d} \gamma_{s s}}{N_{0} B}\right)}_{\mathcal{B}_{1}}\} \\
& +\mathcal{P}\left(\mathcal{H}_{1}\right) B \mathbb{E}\{\underbrace{\log _{2}\left(1+\frac{P_{s}^{u n d} \gamma_{s s}}{N_{0} B+P_{p} \gamma_{p s}}\right)}_{\mathcal{B}_{2}}\} .
\end{aligned}
$$

Expectation $\mathbb{E}\left\{\mathcal{B}_{1}\right\}$, appearing in the first term of (12), is expressed in (13) with $\mathcal{P}\left\{\frac{\mathcal{I}}{\gamma_{s p}} \geq P_{s}\right\}=1-e^{-\frac{\mathcal{I}}{P_{s} \sigma_{s p}^{2}}}$.

For the computation of double integral $J_{1}$, appearing in (13), we have, after some mathematical manipulations and by using $[15,5.1 .28]$

$$
J_{1}=\frac{1}{\ln (2)}\left(J_{1,1}-J_{1,2}\right)
$$

where

$$
\begin{aligned}
J_{1,1} & =e^{-\frac{\mathcal{I}}{P_{s} \sigma_{s p}^{2}}} \ln \left(\frac{N_{0} B \mathcal{I}}{P_{s}}\right)+e^{\frac{1}{P_{s}}\left(\frac{N_{0} B}{\sigma_{s s}^{2}}-\frac{\mathcal{I}}{\sigma_{s p}^{2}}\right)} E_{1}\left(\frac{N_{0} B}{P_{s} \sigma_{s s}^{2}}\right) \\
& +\left(\frac{N_{0} B \sigma_{s p}^{2}}{N_{0} B \sigma_{s p}^{2}-\mathcal{I} \sigma_{s s}^{2}}\right)\left(E_{1}\left(\frac{\mathcal{I}}{P_{s} \sigma_{s p}^{2}}\right)\right. \\
& \left.-e^{\frac{N_{0} B \sigma_{s p}^{2}-\mathcal{I} \sigma_{s s}^{2}}{P_{s} \sigma_{s p}^{2} \sigma_{s s}^{2}}} E_{1}\left(\frac{N_{0} B}{P_{s} \sigma_{s s}^{2}}\right)\right) .
\end{aligned}
$$

Term $J_{1,2}$ is given by

$$
J_{1,2}=\ln \left(\frac{N_{0} B \mathcal{I}}{P_{s}}\right) e^{-\frac{\mathcal{I}}{P_{s} \sigma_{s p}^{2}}}+E_{1}\left(\frac{\mathcal{I}}{P_{s} \sigma_{s p}^{2}}\right) .
$$

The computation of integral $J_{2}$ gives

$$
J_{2}=\frac{1}{\ln (2)} e^{\frac{N_{0} B}{P_{s} \sigma_{s s}^{2}}} E_{1}\left(\frac{N_{0} B}{P_{s} \sigma_{s s}^{2}}\right) .
$$

Expectation $\mathbb{E}\left\{\mathcal{B}_{2}\right\}$, appearing in the second term of (12), is expressed in (18). Thus, multiple integrals $J_{3}$ and $J_{4}$ need to be computed. For integral $J_{3}$ we obtain

$$
J_{3}=\frac{1}{\ln (2)} \int_{0}^{\infty} \frac{e^{\frac{-z}{\bar{z}}}}{\bar{z}} \int_{0}^{\infty} \frac{e^{\frac{-x}{\bar{x}}}}{\bar{x}} J_{3,1} d x d z
$$

where $\bar{x}=\sigma_{s s}^{2}, \bar{z}=\sigma_{p s}^{2}$ and $J_{3,1}$ represents the inner integral of $J_{3}$, that is

$$
J_{3,1}=\int_{u}^{\infty} \ln \left(1+\frac{\mathcal{I} x}{y\left(N_{0} B+P_{p} z\right)}\right) \frac{e^{\frac{-y}{\bar{y}}}}{\bar{y}} d y
$$

where $\bar{y}=\sigma_{s p}^{2}$ and $u=\frac{\mathcal{I}}{P_{s}}$. The latter integral can be computed using integration by parts and exploiting expression [18, 3.352.2]. Then one obtains

$$
\begin{gathered}
J_{3,1}(x, z)=e^{\frac{-u}{\bar{y}}} \ln \left(1+\frac{\mathcal{I} x}{u\left(N_{0} B+P_{p} z\right)}\right)+e^{\frac{\mathcal{I} x}{\bar{y}\left(N_{0} B+P_{p} z\right)}} \times \\
E_{1}\left(\frac{u}{\bar{y}}+\frac{\mathcal{I} x}{\bar{y}\left(N_{0} B+P_{p} z\right)}\right)-E_{1}\left(\frac{u}{\bar{y}}\right) .
\end{gathered}
$$

As a result, by substituting (21) to (19), and by properly transforming variables $x$ and $z$, since a closed form expression of the double integral cannot be derived, two dimensional numerical integration can be applied by employing twice, one for each dimension, the well known Laguerre quadrature rules $[15,25.4 .45]$.

We also need to compute integral $J_{4}$, which is equal to $\mathbb{E}\left\{\mathcal{A}_{2}\right\}$, thus we obtain

$$
\begin{aligned}
& J_{4}=\frac{1}{\ln (2)}\left(\frac{\bar{x}_{1}}{\bar{x}_{1}-\bar{x}_{2}}\right) e^{\frac{N_{0} B}{\bar{x}_{1}}} \times \\
& \\
& \quad\left(E_{1}\left(\frac{N_{0} B}{\bar{x}_{1}}\right)-e^{N_{0} B\left(\frac{\bar{x}_{1}-\bar{x}_{2}}{\bar{x}_{1} \bar{x}_{2}}\right)} E_{1}\left(\frac{N_{0} B}{\bar{x}_{2}}\right)\right) .
\end{aligned}
$$

In the following section, suitable criteria for a fair comparison between the two abovementioned CRN approaches will be studied.

\section{ENSURING FAIRNESS IN THE COMPARISON OF THE TWO CRN APPROACHES}

In this section, the generic design parameters for each of the two CRN approaches will be optimized in the sense of maximizing the ergodic capacity of SU, subject to a QoS outage probability constraint for primary communication.

\section{A. Determining generic parameters of interweaved approach}

Focusing on the interweaved approach, the optimization problem that has to be solved is the following

$$
\begin{aligned}
& \left(\epsilon^{*}, \tau^{*}\right)=\underset{\epsilon, \tau}{\arg \max } \mathbb{E}\left\{R_{s}^{\text {int }}\right\} \\
& \text { s.t. } \mathcal{P}_{\text {out }}^{\text {int }}=\mathcal{P}_{o}, \quad 0 \leq \tau \leq T, \quad \epsilon \geq 0,
\end{aligned}
$$

where, concerning the outage probability constraint, bound $\mathcal{P}_{d, B}$ is used in (6). Taking the outage probability constraint of (23), one obtains the following expression that relates parameters $\epsilon$ and $\tau$.

$$
\epsilon=m_{1}\left(\frac{\kappa}{\sqrt{\tau f_{s}}}+1\right)
$$

where $m_{1}, \kappa$ are quantities equal to $P_{p} \sigma_{00}^{2}+N_{0}$ and $\mathcal{Q}^{-1}\left(\frac{\left(\alpha_{1}+\alpha_{2}\right)\left(1-\mathcal{P}_{o}\right) e^{\frac{\zeta_{0} N_{0} B}{\alpha_{1}}}-\alpha_{1}}{\alpha_{2}}\right)$ respectively, and $\mathcal{Q}^{-1}(\cdot)$ is the inverse of $\mathcal{Q}$-function, which has been already defined. As a result, problem (23) will be expressed as follows

$$
\begin{aligned}
& \left(\epsilon^{*}, \tau^{*}\right)=\underset{\epsilon, \tau}{\arg \max } \mathbb{E}\left\{R_{s}^{\text {int }}\right\} \\
& \text { s.t. } \epsilon=m_{1}\left(\frac{\kappa}{\sqrt{\tau f_{s}}}+1\right), \quad 0 \leq \tau \leq T, \quad \epsilon \geq 0 .
\end{aligned}
$$




$$
\mathbb{E}\left\{\mathcal{B}_{1}\right\}=\underbrace{\int_{0}^{\infty} \int_{\frac{\mathcal{I}}{P_{s}}}^{\infty} \log _{2}\left(1+\frac{\frac{\mathcal{I}}{\gamma_{s p}} \gamma_{s s}}{N_{0} B}\right) f_{\gamma_{s s}}\left(\gamma_{s s}\right) f_{\gamma_{s p}}\left(\gamma_{s p}\right) d \gamma_{s p} d \gamma_{s s}}_{J_{1}}+\mathcal{P}\left\{\frac{\mathcal{I}}{\gamma_{s p}} \geq P_{s}\right\} \underbrace{\int_{0}^{\infty} \log _{2}\left(1+\frac{P_{s} \gamma_{s s}}{N_{0} B}\right) f_{\gamma_{s s}}\left(\gamma_{s s}\right) d \gamma_{s s}}_{J_{2}}
$$

$$
\begin{aligned}
\mathbb{E}\left\{\mathcal{B}_{2}\right\} & =\underbrace{\int_{0}^{\infty} \int_{0}^{\infty} \int_{\frac{\mathcal{I}}{P_{s}}}^{\infty} \log _{2}\left(1+\frac{\frac{\mathcal{I}}{\gamma_{s p}} \gamma_{s s}}{N_{0} B+P_{p} \gamma_{p s}}\right) f_{\gamma_{s s}}\left(\gamma_{s s}\right) f_{\gamma_{s p}}\left(\gamma_{s p}\right) f_{\gamma_{p s}}\left(\gamma_{p s}\right) d \gamma_{s p} d \gamma_{s s} d \gamma_{p s}}_{J_{3}} \\
& +\mathcal{P}\left\{\frac{\mathcal{I}}{\gamma_{s p}} \geq P_{s}\right\} \underbrace{\int_{0}^{\infty} \int_{0}^{\infty} \log _{2}\left(1+\frac{P_{s} \gamma_{s s}}{N_{0} B+P_{p} \gamma_{p s}}\right) f_{\gamma_{s s}}\left(\gamma_{s s}\right) f_{\gamma_{p s}}\left(\gamma_{p s}\right) d \gamma_{s s} d \gamma_{p s}}_{J_{4}}
\end{aligned}
$$

With the aim of solving problem (25), the following proposition can be proved.

Proposition 3. Function $U(\tau)=\mathbb{E}\left\{R_{s}^{\text {int }}\right\}(\tau)$, which is obtained by substituting the outage probability constraint to the objective function of (25) is concave for $\tau \in[0, T]$.

Proof. A detailed proof can be found in [17].

Capitalizing on proposition 3, problem (25) can be solved by applying a gradient ascent method, which is described in algorithm 1 .

\section{Algorithm 1 Optimize $\epsilon$ and $\tau$ for a given $\mathcal{P}_{o}$}

1 Initialization $(n=0)$. Select a $\tau_{0} \in[0, T]$ and increase counter by one.

2 For the $n$-th iteration, compute value $\tau_{n}$ as follows

$$
\tau_{n}=\tau_{n-1}+\left.\lambda \frac{\partial U(\tau)}{\partial \tau}\right|_{\tau=\tau_{n-1}}
$$

where $\lambda$ stands for the step of the algorithm.

3 Increase counter $n$ by one and if $n$ overcomes a maximum number of iterations, stop, otherwise go to step 2 .

4 Having found $\tau^{*}$ compute the corresponding

$$
\epsilon^{*}=m_{1}\left(\frac{\kappa}{\sqrt{\tau^{*} f_{s}}}+1\right) \text {. }
$$

\section{B. Determining generic parameters of underlay approach}

In the underlay case, the interference temperature, $\mathcal{I}^{*}$, corresponding to the same examined outage level, $\mathcal{P}_{o}$, can be found by setting $\mathcal{P}_{\text {out }}^{\text {und }}=\mathcal{P}_{o}$, leading to equation $g\left(\mathcal{I}^{*}\right)=0$, where

$$
g(\mathcal{I})=\mathcal{P}_{\text {out }}^{\text {und }}-\mathcal{P}_{o}
$$

and the expression of $\mathcal{P}_{\text {out }}^{\text {und }}$ is given in (11). As the outage probability of $\mathrm{PU}$ is monotonically increasing with $\mathcal{I}$, one can find that there is a single $\mathcal{I}^{*} \geq 0$ to search for and this can be done by applying Newton's method.

In the following section, the throughput performance of the two examined CRN approaches will be evaluated.

\section{Numerical Evaluation}

With the aim of evaluating the performance of the two examined CRN approaches, extensive Monte Carlo (MC) simulations have been performed in order to confirm the validity of the theoretical expressions derived. More specifically, 5000 MAC frames were simulated. According to the scenario, the SNR levels of the involved channels are $\sigma_{p p}^{2}=\sigma_{s s}^{2}=20 \mathrm{~dB}$ for the direct links, $\sigma_{p s}^{2}=\sigma_{s p}^{2}=18 d B$ for the cross-links and $\sigma_{00}^{2}=6 d B$ for the link between $B S_{p}$ and $B S_{s}$. Moreover, we set $B=1 \mathrm{~Hz}, f_{s}=6 \mathrm{MHz}, T=100 \mathrm{~ms}$ and also unit noise variance is assumed. In addition, the SINR level of the PU, below which an outage occurs is set to be $\zeta_{0}=0 \mathrm{~dB}$.

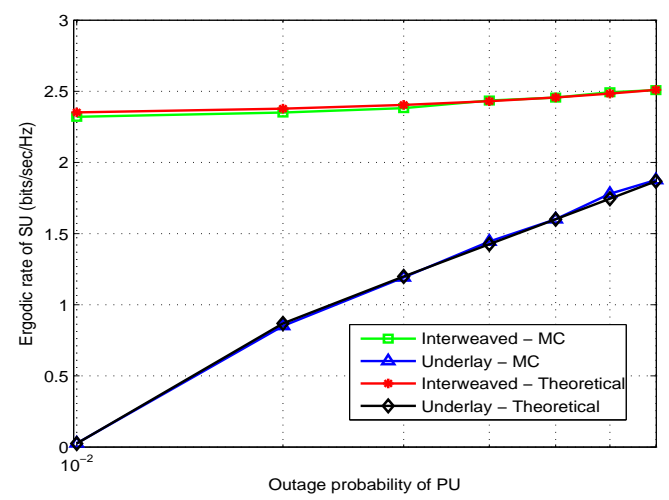

Fig. 3. Ergodic SU capacity vs. outage probability at $\mathrm{PU}, \mathcal{P}\left(\mathcal{H}_{1}\right)=0.6$.

In Fig. 3 the ergodic rate of the SU, considering an interweaved $\mathrm{CRN}$, is depicted as a function of the outage probability of the PU and compared with the ergodic rate obtained at the SU when the underlay approach is adopted. Both MC and theoretical curves are depicted. In the examined scenario, the primary system is active with probability $\mathcal{P}\left(\mathcal{H}_{1}\right)=$ 0.6. The curves shown demonstrate a clear capacity gain in favour of the interweaved approach for all examined levels of PU outage probability.

In Fig. 4, the same performance metric is depicted with the only difference lying in the fact that the activity of the primary system is denser $\left(\mathcal{P}\left(\mathcal{H}_{1}\right)=0.96\right)$. Here, one can 


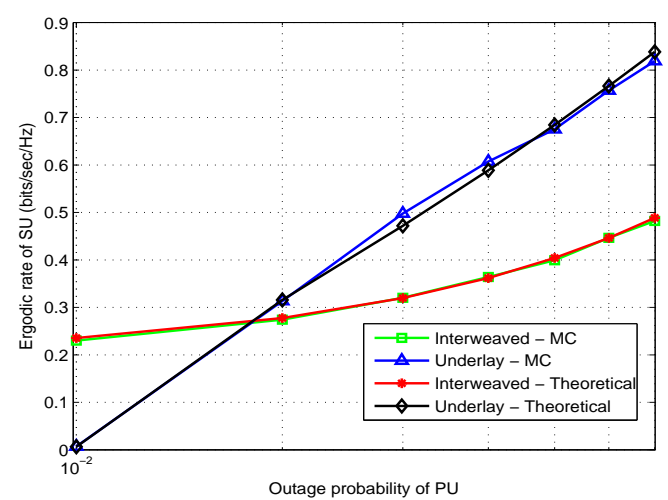

Fig. 4. Ergodic SU capacity vs. outage probability at $\mathrm{PU}, \mathcal{P}\left(\mathcal{H}_{1}\right)=0.96$.

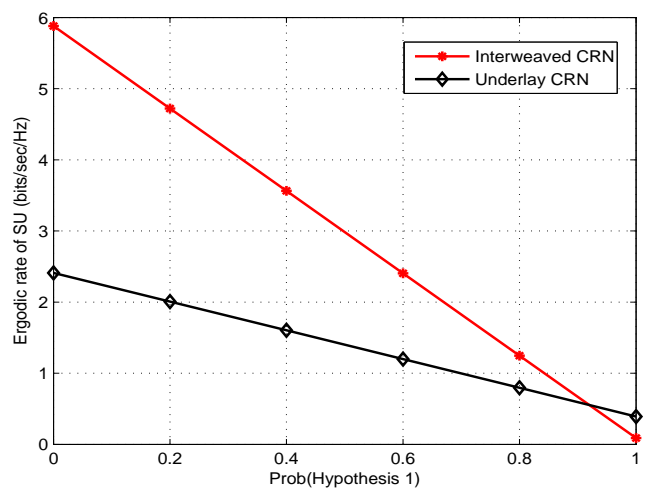

Fig. 5. Ergodic SU capacity vs. primary system's activity profile, target PU outage probability $\mathcal{P}_{o}=0.03$.

observe that the throughput gain in favour of the interweaved CRN approach has been significantly reduced and when the target PU outage probability becomes higher than $2 \%$, the throughput of the underlay CRN approach outperforms the one of the interweaved approach.

Finally, in Fig. 5, the same metric is depicted as a function of primary system's activity rate when the target PU outage probability is $3 \%$. One can observe that, regardless of the CRN approach followed, the ergodic capacity of SU is a decreasing function of the activity of primary communication, which can be justified because as this activity becomes denser, the average interference (over time) received at SU increases. Additionally, the throughput of the interweaved CRN approach overcomes the one of the underlay approach for primary systems which are active for up to $90 \%$ of the time.

\section{CONCLUSIONS}

In this paper, the interweaved CRN approach was examined and compared with the underlay CRN approach in terms of the ergodic throughput of the SU for a common PU outage level. Expressions for the ergodic capacity of the SU as well as for the outage probability of primary communication were derived for both approaches and it was numerically shown that the comparison results depend on the activity profile of primary communication. A future extension will also elaborate on the quality of spectrum sensing, by considering systems the transmitters of which are equipped with multiple antennas.

\section{ACKNOWLEDGMENT}

This work was supported by the French national ANRVERSO funded project LICORNE. Dr. Ropokis was funded by the MIMOCORD project. ${ }^{1}$

\section{REFERENCES}

[1] E. Biglieri, A. J. Goldsmith, L. J. Greenstein, N. Mandayam, and H. V. Poor, Principles of Cognitive Radio. Cambridge University Press, 2012

[2] S. Haykin, "Cognitive radio: brain-empowered wireless communications," IEEE Journal on Selected Areas in Communications, vol. 23, no. 2, pp. 201-220, 2005.

[3] A. Goldsmith, S. Jafar, I. Maric, and S. Srinivasa, "Breaking spectrum gridlock with cognitive radios: An information theoretic perspective," Proceedings of the IEEE, vol. 97, no. 5, pp. 894-914, 2009.

[4] S. Srinivasa and S. Jafar, "The throughput potential of cognitive radio: A theoretical perspective," in Fortieth Asilomar Conference on Signals, Systems and Computers, 2006. ACSSC '06, 2006, pp. 221-225.

[5] A. Giorgetti, M. Varrella, and M. Chiani, "Analysis and performance comparison of different cognitive radio algorithms," in $\operatorname{Cog} A R T, 2009$, pp. $127-131$

[6] M. Khoshkholgh, K. Navaie, and H. Yanikomeroglu, "Access strategies for spectrum sharing in fading environment: Overlay, underlay, and mixed," IEEE Transactions on Mobile Computing, vol. 9, no. 12, pp. 1780-1793, 2010.

[7] X. Kang, Y.-C. Liang, H. Garg, and L. Zhang, "Sensing-based spectrum sharing in cognitive radio networks," IEEE Transactions on Vehicular Technology, vol. 58, no. 8, pp. 4649-4654, 2009.

[8] Y. Wang, P. Ren, F. Gao, and Z. Su, "A hybrid underlay/overlay transmission mode for cognitive radio networks with statistical quality-of-service provisioning," IEEE Transactions on Wireless Communications, vol. PP, no. 99, pp. 1-17, 2014.

[9] A. Ghasemi and E. Sousa, "Fundamental limits of spectrum-sharing in fading environments," IEEE Transactions on Wireless Communications, vol. 6, no. 2, pp. 649-658, 2007

[10] C.-X. Wang, X. Hong, H.-H. Chen, and J. Thompson, "On capacity of cognitive radio networks with average interference power constraints," IEEE Transactions on Wireless Communications, vol. 8, no. 4, pp. 1620$1625,2009$.

[11] L. Musavian and S. Aissa, "Ergodic and outage capacities of spectrumsharing systems in fading channels," in IEEE Global Telecommunications Conference, 2007. GLOBECOM '07, 2007, pp. 3327-3331.

[12] L. Sboui, Z. Rezki, and M.-S. Alouini, "A unified framework for the ergodic capacity of spectrum sharing cognitive radio systems," IEEE Transactions on Wireless Communications, vol. 12, no. 2, pp. 877-887, 2013.

[13] H. Suraweera, P. Smith, and M. Shafi, "Capacity limits and performance analysis of cognitive radio with imperfect channel knowledge," IEEE Transactions on Vehicular Technology, vol. 59, no. 4, pp. 1811-1822, 2010.

[14] S. Stotas and A. Nallanathan, "On the outage capacity of sensingenhanced spectrum sharing cognitive radio systems in fading channels," IEEE Transactions on Communications, vol. 59, no. 10, pp. 2871-2882, 2011.

[15] M. Abramowitz and I. A. Stegun, Handbook of mathematical functions. Dover publications, 1965.

[16] Y.-C. Liang, Y. Zeng, E. Peh, and A. T. Hoang, "Sensing-throughput tradeoff for cognitive radio networks," IEEE Transactions on Wireless Communications, vol. 7, no. 4, pp. 1326-1337, 2008.

[17] M. C. Filippou, D. Gesbert, and G. A. Ropokis, "On the throughput comparison between interweaved and underlay cognitive radio networks," 2014. [Online]. Available: https://sites.google.com/site/miltosfilippou/downloads

[18] I. S. Gradshteyn and I. M. Ryzhik, Table of integrals, series, and products, 7th ed. Elsevier/Academic Press, Amsterdam, 2007.

1 The project is implemented within the framework of the Action "Supporting Postdoctoral Researchers" of the
Operational Program "Education and Lifelong Learning" (Action's Beneficiary: General Secretariat for Research and Operational Program "Education and Lifelong Learning" (Action's Beneficiary: Gener
Technology), and is co-financed by the European Social Fund (ESF) and the Greek State. 